\title{
Psychosocial Development and First Substance Use in Third and Fourth Grade Students: A Short-Term Longitudinal Study
}

\author{
Randall M. Jones \\ Family, Consumer, and Human Development, Utah State University, Logan, UT 84322-2905, USA \\ Correspondence should be addressed to Randall M. Jones, r.jones@usu.edu
}

Received 25 August 2010; Revised 27 January 2011; Accepted 24 February 2011

Academic Editor: Jeffrey W. Fagen

Copyright () 2011 Randall M. Jones. This is an open access article distributed under the Creative Commons Attribution License, which permits unrestricted use, distribution, and reproduction in any medium, provided the original work is properly cited.

Third and fourth grade students $(n=509)$ completed measures of psychosocial development, substance use, and intentions to use in January, and again in May, 1998. A revised Erikson Psychosocial Inventory Scale (EPSI) was employed to assess psychosocial development while estimates of substance use and intentions were obtained from anonymous self-reports. The sample was split on the basis of change in substance use and intentions from January to May. Using this grouping scheme as an independent variable, scores on the EPSI subscales (trust, autonomy, initiative, industry, and identity) were compared over time. Interestingly, no differences in psychosocial development were evident for the initial substance use comparisons, but differences were evident five months later. Respondents who initiated substance use and/or increased intentions during the five-month interval exhibited small gains (averaging less than 2.0\%) on measures of trust, autonomy, and initiative and modest declines in industry and identity $(-1.2 \%)$. Respondents who neither initiated substance use nor increased intentions during the five-month interval experienced significant gains (averaging 6.0\%) on all five of the EPSI subscales. These findings suggest that early substance use may impede psychosocial development, thus justifying prevention efforts in the earlier grades as well as efforts to delay onset.

\section{Introduction}

Risk and resiliency has spawned much research over the past four decades. Much of this work has focused upon identifying characteristics that contribute to "risk" such as low socioeconomic status, alcoholic and/or psychotic parents, maternal employment, and troubled family relationships, marked by separation and/or divorce, which are shared among adolescents who exhibit problem behaviors including school drop-out, substance use and abuse $[1,2]$, precocious and risky sexual behaviors, and delinquency. Tangentially, a more positive approach has emerged amidst a growing number of studies that have opted to view these problem behaviors somewhat differently by examining groups of "at risk" children and documenting "resiliency" factors that appear to buffer high risk children against seemingly probable doom.

The literature on resilient children [3] echoes Erikson's $[4,5]$ theoretical guidelines regarding psychosocial stages of human development. For example, Erikson [4-6] contends that a central psychosocial task during the early years, that is, the first task in personality development, involves balancing trust and mistrust. Erikson believes that resolution of this stage is influenced by the relationship between a primary caregiver and her child, and, thus, mothers create a sense of trust in their children [6, page 63]. Werner [7] provides empirical support for Erikson's contention by observing that the resilient children in her study had received a great deal of attention from their primary caretakers during the first year of life, especially when compared to children who later developed serious coping problems. Werner's resilient children had "at least one caregiver from whom they received lots of attention during the first year of life" [3, page 69]. Resilient children were in situations that, according to Erikson, should promote a healthy balance of trust over mistrust.

The second stage in Erikson's theory of lifespan development (autonomy versus shame/doubt) is paramount during the second and third years of life when the child begins to recognize that they are individuals, somewhat distinct from their primary caregivers [4]. Werner observed that resilient children exhibit "pronounced autonomy" [8] who 
seek out novel experiences, lack fear, and are quite self-reliant [3].

Erikson's third stage (initiative versus guilt) is apparent in the behavior of four- and five-year-old children who possess a "surplus of energy which permits him to forget failures quickly and to approach what seems desirable (even if it also seems dangerous) with undiminished and better aimed effort" [6, page 75]. These same qualities characterize resilient children who often seek refuge in hobbies and creative interests [3]. According to Werner, "such activities, whether it was fishing, swimming, horseback riding, or hula dancing, gave them a reason to feel proud. Their hobbies, and their lively sense of humor, became a solace when things fell apart" [3, page 69].

A defining characteristic of stage four (industry versus inferiority) is the child's desire to be shown how to work with others [6]. Children grappling with industry and inferiority will either feel competent as they learn and do well, or inferior as they fail. Werner noted that resilient children and adolescents seek opportunities to be helpful such as caring for younger siblings, managing the household, to assist parents, working part time to contribute needed resources, and engaging in school activities, both curricular and extracurricular [3].

Clearly, the psychological and behavioral characteristics observed in resilient children by Werner and others echo Erikson's script for healthy psychosocial development.

For Erikson [4-6], successful resolution of the first four developmental stages is necessary for resolving identity issues during adolescence and young adulthood. These early psychosocial stages, left unresolved, will likely impede identity development as well as resolution of Erikson's subsequent developmental stages during adulthood (stages six through eight).

Most research associated with Erikson's fifth stage has employed Marcia's [9] identity status paradigm which defines four identity statuses in terms of crisis or exploration, and commitment. Marcia's paradigm has generated a wealth of research, most generally supportive of Erikson's developmental trajectory. The identity statuses have also yielded strong and consistent relationships with substance use and abuse among adolescents and young adults. Jones and Hartmann [10] surveyed 12,500+ adolescents and found that diffused respondents (low commitment, low exploration) were more likely to report involvement with substances when compared to the achieved (commitment following a period of exploration), moratorium (high exploration and low commitment), and foreclosed respondents (high commitment, low exploration), while foreclosures were least likely to have engaged in licit and illicit substance use. Indeed, diffused adolescents were twice as likely to have tried cigarettes and alcohol, three times as likely to have tried marijuana, four times as likely to have tried inhalants, and five times as likely to have used cocaine when compared to their foreclosed peers. Without exception, the moratorium and achieved adolescents reported substance use experience that fell between these extremes.

In a related effort, the identity status construct was employed to discriminate known substance abusers (i.e., those attending a two-year residential program for substance abuse) from adolescents attending public schools. The public school sample was matched to the clinical sample on several demographic characteristics (age, gender, ethnicity, religiosity, etc.). Jones et al. [11] reported that all comparisons reflected lower psychosocial maturity among the clinical group as compared to student comparison group (specifically, lower scores on the measures of achievement and moratorium, higher scores on the measure of foreclosure). Using identity profiles in a discriminant analysis, Jones et al. [11] were able to classify all but one (98.2\%) of 54 respondents (27 clinical and 27 nonclinical) correctly into the clinical and nonclinical groups.

Finally, Christopherson et al. [12] found significant motivational (related to substance use) differences among adolescents across the identity statuses. Respondents classified as either achieved or in moratorium were much more likely to cite curiosity and recreation as reasons for using substances as compared to their foreclosed and diffused peers, who cited peer pressure and boredom as reasons for using substances. Differences were also evident in reasons for not using substances. Diffused respondents were most likely to cite fear of parents finding out, and fear of arrest as reasons for not trying alcohol, whereas the achieved and moratorium youths were simply not interested, or expressed health concerns.

Given that (1) resilient children likely possess developmental qualities (namely; trust, autonomy, initiative, and industry) that are necessary for healthy resolution of the identity crisis, and (2) diffused adolescents (those who have not successfully resolved the identity "crisis") report higher levels of substance use and abuse, the issue of temporal ordering becomes paramount. On the one hand, the literature on resiliency implies that children who are psychosocially healthy may be inoculated against the pressures involved with substance use and associated at risk behaviors; and similarly, research on identity and substance use has shown that adolescents who are psychosocially healthy are less likely to use and abuse substances. Does early involvement with substances impede healthy psychosocial development? This short-term longitudinal study was conceived to address these issues.

\section{Methods}

2.1. Sample. The data for this study was obtained from extant data that was collected to evaluate substance use/abuse prevention efforts with a single school district in Southwest Arizona during the 1998 school year. All students within the district were invited to participate in the evaluation effort by completing questionnaires in January and again in May. Of relevance to this study on psychosocial development and substance use were the data provided by 509 (grade $3: 231$; grade $4: 278$ ) children, attending the four elementary schools within the district and who participated in both assessments (January and May). Of these, 51.9\% were male, and a majority Anglo (77.4\%; 2.6\% Black; 0.4\% Oriental; 13.0\% Hispanic; $2.6 \%$ American Indian; 0.4\% Filipino; 3.8\% 
other). Most (75.4\%) reported that they were living with both biological parents (17.5\% divorced; $2.9 \%$ separated; $2.2 \%$ paternal loss due to death; $0.2 \%$ maternal loss due to death; $1.8 \%$ did not respond to this question).

2.2. Measurement. A questionnaire was employed to evaluate substance use prevention efforts within the participating school district. The questionnaire contains questions that assess demographic characteristics such as age, grade, ethnicity, and parents marital status; six questions about previous substance use and six questions that measure intentions to use; 60 items that measure Erikson's first five developmental stages (12 each for Trust, Autonomy, Initiative, Industry, and Identity).

Substance Use. Substance Use was measured with six nominal response questions (yes or no) that asked whether or not the respondents had ever tried cigarettes, smokeless tobacco, alcohol, marijuana, inhalants, and cocaine. (Example: have you ever tried smoking a cigarette? Yes/No.) Since each of these six questions represents a single-item indicator, no attempt was made to examine reliability and validity.

Intentions to Use. Intentions to Use was measured with six nominal response questions (yes or no) that asked about intentions to use cigarettes, smokeless tobacco, alcohol, marijuana, inhalants, and cocaine. (Example: if you have never tried smoking a cigarette, do you believe you ever will? Yes/No). Each question was a single-item indicator.

Erikson's Stages of Development. Erikson's stages of development were assessed using a modified version of the Rosenthal et al. [13] Erikson Psychosocial Inventory Scale (EPSI). The EPSI was developed to measure the first six stages of Erikson's eight-stage developmental theory (Trust, Autonomy, Initiative, Industry, Identity, and Intimacy) among "respondents of about 13 years of age or above" [13, page 528]. Given the youth of these participants ( $3 \mathrm{rd}$ and 4 th grade), some of the original EPSI items were modified. Changes to the EPSI were informed by several third and fourth grade teachers, one elementary school principal, and two volunteer parents (who, at the time, had children in either the third or fourth grades). Most of these changes were minor and involved replacing one or two words to simplify vocabulary.

Of the 12 original Trust (versus Mistrust) items, nine were retained in their original form, two items were modified slightly, and one item was replaced completely; for the 12 original Autonomy (versus Shame and Doubt) items, six remained the same, five were modified, and one was replaced; for the 12 original Initiative (versus Guilt) items, ten remained the same, and two were replaced; for the 12 original Industry (versus Inferiority) items, six remained the same, five were modified, and one was replaced; for the 12 original Identity (versus Identity Confusion) items, seven were unchanged, four underwent slight revision, and one was rewritten completely. An additional modification involved changing the response format from a 5-point Likert response format to a simple dichotomy ("Almost Always," "Almost Never"). Finally, the original EPSI contains 12 items that measure Erikson's sixth stage of psychosocial development (Intimacy versus Isolation). All 12 of these items were deemed inappropriate for 3rd and 4th grade students, and hence, the 12 intimacy items were not included in the revised EPSI.

2.3. Procedures. Classroom teachers administered questionnaires to all students in early January, $1998(n=697)$, and again in late May, 1998 ( $n=706)$. Time- 1 / time- 2 matches were obtained for 509 students. For both administrations, classroom teachers helped students with completion of the demographic questions, read each question aloud, and waited for students to mark their answer forms. Due to the length of the instrument and the youth of these participants, assessment was lagged over two consecutive days with each session lasting one hour.

\section{Results}

Reliability estimates and interscale correlations were generated to ensure that the revised EPSI subscales (combined with administration procedures and the youth of this sample) yielded psychometric properties comparable to those of the original scales. As shown in Table 1, all five subscales yielded moderate levels of internal consistency with estimates ranging from a low of $\mathrm{KR}-20=.56$ (Initiative), to a high of $\mathrm{KR}-20=.67$ (Trust and Industry). These estimates are comparable to those in the original study [13] where Cronbach Alpha coefficients ranged from.57 (Initiative) to .75 (Industry). Likewise, interscale correlations generated for the revised EPSI are consistent with those in the Rosenthal study [13]. The largest bivariate correlations (.60) were obtained between Trust and Initiative, and Trust with Identity. Rosenthal et al. [13] reported correlations of.50 and.41 for these comparisons. The coefficients presented in Table 1 indicate that our revisions to the EPSI yielded psychometric properties (internal consistency and convergent validity) that mirror those of the original EPSI.

Paired $t$-tests were calculated for each of the five EPSI subscales to examine psychosocial development over the fivemonth interval between the first and second assessments. Statistically significant increases were observed on all five of the subscales: Trust $M 1=8.09, M 2=8.65, t(507)=$ 5.22, and $P<.05$; Autonomy $M 1=9.46, M 2=10.06$, $t(507)=6.57$, and $P<.05$; Initiative $M 1=8.65$, $M 2=9.05, t(507)=4.23$, and $P<.05$; Industry $M 1=8.16, M 2=8.53, t(507)=3.76$, and $P<.05$; Identity $M 1=9.08, M 2=9.47, t(507)=4.07$, and $P<$ .05. These comparisons support Erikson's beliefs concerning psychosocial development by demonstrating developmental advances over time, thus providing evidence of construct validity for the revised EPSI. Increases ranged from a low of $4.3 \%$ (Identity) to a high of $6.9 \%$ (Trust).

Results from these preliminary analyses support use of the revised EPSI with younger samples. And, because interscale correlations are all quite large-and positive (cf., 
TABLE 1: Estimates of internal consistency and interscale correlations for the subscales of the revised Eriksonian Psychosocial Scale Inventory (EPSI).

\begin{tabular}{|c|c|c|c|c|c|}
\hline & \multicolumn{5}{|c|}{ Revised EPSI subscales } \\
\hline & Trust & Autonomy & Initiative & Industry & Identity \\
\hline (1) Trust & $(67 / 73)$ & .55 & .60 & .54 & .60 \\
\hline (2) Autonomy & .70 & $(61 / 70)$ & .57 & .50 & .56 \\
\hline (3) Initiative & .67 & .68 & $(56 / 64)$ & .58 & .58 \\
\hline (4) Industry & .68 & .64 & .64 & $(67 / 69)$ & .51 \\
\hline (5) Identity & .69 & .71 & .66 & .64 & $(61 / 71)$ \\
\hline
\end{tabular}

Diagonal entries are KR-20 coefficients (1st assessment/2nd assessment); off-diagonal entries are Pearson correlation coefficients. (Coefficients above the diagonal emerged from 1st assessment, those below from time 2. )

Table 1)—the revised EPSI is subject to the same criticism as the original version.

Table 2 summarizes substance use experience and intentions for both assessment occasions. As shown, experience increased for all substances during the five-month interval, and these increases were statistically significant for smokeless tobacco, marijuana, and cocaine. Likewise, intentions to use increased for all substances. These increases were statistically significant for cigarettes, inhalants, and alcohol.

Participants were grouped on the basis of whether or not initial substance use and intentions occurred during the five-month interval, or if abstinence was maintained during this time frame. The following procedures were used to create two groups. Two questions were asked for each of six substances.

(1) Have you ever tried $X$ ?

(2) If not, do you think you will ever try X?

If a respondent answered Yes to question 1, they received 1 point (and did not answer question 2). If a respondent answered No to question 1 and Yes to question 2, they received 1 point. If a respondent answered No to question 1 and No to question 2, they received 0 points.

Responses to all twelve questions ( 1 experience and 1 intention for each of six substances) were summed separately for each assessment occasion, thus yielding a range from 0-6 for the January assessment, and 0-6 for the May assessment. January scores were subtracted from May scores and differences ranged from 0-6 (i.e., none of these differences were negative indicating that no participant exhibited a decrease in use/intentions). All respondents who reported initial use of at least one new substance, or an increase in intentions between January and May were combined into an "increase" experience/intentions group $(n=131)$; respondents who reported no use and no intentions in both January and May constituted the "no use" group $(n=378)$. Since the largest experience/intention increase was observed for alcohol (cf., Table 2), and three of the five remaining substances yielded increases less than $2.0 \%$, no attempt was made to form separate groups for each type of substance.

In order to examine the relationship between psychosocial development and substance use experience, a $2 \times 2 \times 2$ multivariate analysis of variance was computed where all five revised EPSI subscales were entered as dependent measures, and grade (3rd versus 4 th), substance experience/intentions (increase versus no use), and gender (male versus female) constituted independent variables. Results from this analysis yielded statistically significant multivariate $F$ ratios for the main effects of Gender $(F[5,495)=2.70 ; P<.05)$ and Substance Experience $(F[5,495]=2.31 ; P<.05)$; but the Grade effect was not statistically significant $(F[5,495]=$ $0.72 ; P>.60$ ). All interactions (2-way: Gender $x$ Substance Experience, Grade $x$ Substance Experience, and Gender $x$ Grade; 3-way: Gender $x$ Substance Experience $x$ Grade) were not statistically significant.

To isolate gender and substance experience effects for each of the five psychosocial measures, post hoc comparisons were generated using separate five ANCOVAs. Specifically, a Gender (2) $x$ Substance Experience (2) $x$ Grade (2) ANCOVA (using time 2 scale scores as dependent measures and the matching time 1 score as a covariate) was computed for each of the five EPSI subscales. For the measure of Trust, the main effect for Substance Experience was statistically significant $(F[1,498]=3.41 ; P<.05)$. Cell means show that respondents who reported initial substance experience/intentions during the 5-month interval (Trust $M=8.30$ ) scored statistically significantly lower than those who did not (Trust $M=8.69)$. Means adjusted for all three independent variables and the covariate were more discrepant ( 8.25 versus 8.69). Main effects for Grade and Gender, as well as the 2- and 3-way interactions were not statistically significant.

When Autonomy scores were entered as the dependent variable, statistically significant effects emerged for the Grade $x$ Gender interaction $(F[1,498]=5.89 ; P<.05)$ only. Cell means revealed that fourth grade males obtained the highest scores on Autonomy $(M=10.26)$, and fourth grade females the lowest $(M=9.60)$. Mean scores for third grade males and females were 9.80 and 10.25 , respectively.

For Initiative, the main effects for Gender, Substance Experience, and Grade failed to yield statistical significance. All 2-way and the 3-way interactions failed to achieve statistical significance as well.

The Industry scale was statistically significantly related to Substance Experience $(F[1,498]=7.75 ; P<.05)$. Cell means revealed that respondents who reported initial substance use and/or intentions to use $(M=8.00)$ scored significantly lower than those who did not $(M=8.62)$.

For Identity scores, significant effects emerged for Substance Experience $(F[1,498]=3.62 ; P<.05)$, and the Grade $x$ Substance Experience interaction $(F[1,498]=4.31$; 
TABLE 2: Change in third and fourth grader substance experience over a five-month interval.

\begin{tabular}{|c|c|c|c|c|}
\hline & January & May & Change & $t$ \\
\hline Have tried cigarettes & $11.2 \%$ & $13.6 \%$ & $+2.4 \%$ & 1.61 \\
\hline Intend to try cigarettes & 6.5 & 9.2 & +2.7 & $2.07^{*}$ \\
\hline Have tried smokeless tobacco & 8.8 & 11.4 & +2.6 & $2.04^{*}$ \\
\hline Intend to try smokeless tobacco & 4.3 & 6.5 & +2.2 & 1.86 \\
\hline Have tried inhalants & 2.8 & 3.3 & +.5 & .60 \\
\hline Intend to try inhalants & 1.4 & 3.1 & +1.7 & $2.07^{*}$ \\
\hline Have tried alcohol & 35.4 & 39.3 & +3.9 & 1.72 \\
\hline Intend to try alcohol & 22.6 & 32.4 & +9.8 & $4.66^{*}$ \\
\hline Have tried marijuana & .5 & 2.0 & +1.5 & $2.12^{*}$ \\
\hline Intend to try marijuana & .6 & 1.6 & +1.0 & 1.67 \\
\hline Have tried cocaine & .2 & 1.4 & +1.2 & $2.13^{*}$ \\
\hline Intend to try cocaine & 1.0 & 2.0 & +1.0 & 1.29 \\
\hline
\end{tabular}

${ }^{*}$ Indicates statistical significance $(P<.05)$.

Table 3: Means and standard deviations depicting the relationship between psychosocial development and initial substance use/intentions over five-months.

\begin{tabular}{|c|c|c|c|c|}
\hline & & $\begin{array}{c}\text { January } \\
M\end{array}$ & $\begin{array}{c}\text { May } \\
M\end{array}$ & $\%$ Change \\
\hline \multicolumn{5}{|l|}{ Increased substance use and/or intentions $(n=131)$} \\
\hline & Trust & 8.21 & 8.30 & $+1.1 \%$ \\
\hline & Autonomy & 9.52 & 9.89 & $+3.9 \%$ \\
\hline & Initiative & 8.73 & 8.83 & $+1.2 \%$ \\
\hline & Industry & 8.17 & 7.99 & $-2.2 \%$ \\
\hline & Identity & 9.13 & 9.12 & $-.1 \%$ \\
\hline \multicolumn{5}{|l|}{ No substance use and/or intentions $(n=378)$} \\
\hline & Trust & 8.06 & 8.70 & $+7.9 \% *$ \\
\hline & Autonomy & 9.44 & 10.04 & $+6.4 \% *$ \\
\hline & Initiative & 8.62 & 9.05 & $+5.0 \% *$ \\
\hline & Industry & 8.16 & 8.63 & $+5.8 \% *$ \\
\hline & Identity & 9.06 & 9.50 & $+4.9 \% *$ \\
\hline
\end{tabular}

$P<.05)$. Respondents who reported initial substance use and/or intentions from January to May obtained a mean Identity score of 9.13, compared to the abstainers at 9.49. The Grade $x$ Substance Experience interaction indicated that fourth grade abstainers scored a mean of 9.41, compared to their peers at 9.52. For third grade respondents, the former obtained a mean of 9.55, and the latter 8.75. All other comparisons failed to yield statistical significance.

\section{Summary}

The main effects of Gender and Grade did not yield statistically significant differences on the revised EPSI subscales (although there was a statistically significant Grade $x$ Gender interaction for Autonomy scores). Substance Experience, however, did yield statistically significant differences on three of the five revised EPSI subscales (namely; Trust, Industry, and Identity) as well as a significant Grade $x$ Substance Experience interaction for Identity. Table 3 summarizes results from the analyses specific to substance use and psychosocial development. In all instances, students who reported initial substance use and/or increased intentions during the five-month interval also scored lower on the measures of psychosocial development when compared to peers who reported no experience and no intentions. These differences were not statistically significant for Autonomy and Initiative; but, comparisons for Trust, Industry, and Identity were quite discrepant. Students who reported initial substance use and/or increased intentions during the 5month interval also exhibited small gains in Trust $(+1.1 \%)$, Autonomy $(+3.9 \%)$, and Initiative $(+1.2 \%)$, and small decreases in Industry $(-2.2 \%)$ and Identity $(-0.1 \%)$ during the five-month interval. Participants who reported no initial substance use and no intentions netted substantial gains on all five psychosocial measures (Trust $+7.9 \%$, Autonomy $+6.4 \%$, Initiative $+5.0 \%$, Industry $+5.8 \%$, and Identity $+4.9 \%)$.

These findings indicate that early involvement with various substances may impede psychosocial development. Hence, the fact that "diffused" adolescents report substantially more substance use [10] is partially explained by 
the possibility that substance use may hamper successful resolution of Erikson's earlier developmental tasks (namely; Trust, Autonomy, Initiative, and Industry) which are fundamental for resolving identity issues, thus inflating the likelihood of identity diffusion and substance use during adolescence. Findings from this study confirm speculation that early substance use/abuse may impede adolescent identity development [14, page 103].

In a similar vein, these findings provide support for prevention programs that are administered early in the lifespan with the goal of delaying onset of substance use and related behaviors. The Department of Health and Human Services asserts that "early experimentation is reported to lead to regular use and, for all too many individuals, to compulsive patterns of use characterized by psychological and physiological dependence" [15, page 35]. The literature on resiliency in conjunction with empirical evidence linking substance use/abuse and psychosocial well-being supports the use of prevention activities that foster psychosocial development as a means of delaying and/or preventing these behaviors.

Findings from this study of 3rd and 4th grade children indicate that the negative effects of substance use/abuse on adolescent development may be manifest in the early stages of psychosocial development. Systematic replication of these findings will further clarify these relations. In the meantime, we need to recognize that the adage "smoking will stunt your growth" may also be applicable to social and psychological development, in addition to somatic development.

\section{References}

[1] R. Jessor, J. A. Chase, and J. E. Donovan, "Psychosocial correlates of marijuana use and problem drinking in a national sample of adolescents," American Journal of Public Health, vol. 70, no. 6, pp. 604-613, 1980.

[2] M. D. Newcomb and P. M. Bentler, Consequences of Adolescent Drug Use: Impact on the Lives of Young Adults, Sage, Newbury Park, Calif, USA, 1988.

[3] E. E. Werner, "Resilient children," Young Children, vol. 4, pp. 68-72, 1984.

[4] E. H. Erikson, Childhood and Society, Norton, New York, NY, USA, 2nd edition, 1963.

[5] E. H. Erikson, Identity: Youth and Crisis, Norton, New York, NY, USA, 1968.

[6] E. H. Erikson, "Growth and crisis of the healthy personality," in Psychological Issues, G. S. Klein, Ed., International Universities Press, New York, NY, USA, 1959.

[7] E. E. Werner, "Resilient offspring of alcoholics: a longitudinal study from birth to age 18," Journal of Studies on Alcohol, vol. 47, no. 1, pp. 34-40, 1986.

[8] L. Murphy and A. Moriarty, Vulnerability, Coping and Growth from Infancy to Adolescence, Yale University Press, New Haven, Conn, USA, 1976.

[9] J. E. Marcia, "Development and validation of ego-identity status," Journal of Personality and Social Psychology, vol. 3, no. 5, pp. 551-558, 1966.

[10] R. M. Jones and B. R. Hartmann, "Developmental differences and experimental substance use among adolescents," Journal of Adolescence, vol. 11, no. 4, pp. 347-360, 1988.
[11] R. M. Jones, B. R. Hartmann, C. O. Grochowski, and P. Glider, "Ego identity and substance abuse: a comparison of adolescents in residential treatment with adolescents in school," Personality and Individual Differences, vol. 10, no. 6, pp. 625-631, 1989.

[12] B. B. Christopherson, R. M. Jones, and A. P. Sales, "Diversity in reported motivations for substance use as a function of egoidentity development," Journal of Adolescent Research, vol. 3, pp. 141-152, 1988.

[13] D. A. Rosenthal, R. M. Gurney, and S. M. Moore, "From trust to intimacy: a new inventory for examining Erikson's stages of psychosocial development," Journal of Youth and Adolescence, vol. 10, no. 6, pp. 525-537, 1981.

[14] D. Baumrind, "A developmental perspective on adolescent risk taknig in contemporary America," in Adolescent Social Behaviorand Health: New Directions for Child Development, C. E. Irwin Jr., Ed., vol. 37, 1987.

[15] DHHS, Drug Abuse and Drug Abuse Research: The First in a Series of Triennial Reports to Congress, Government Printing Office, Washington, DC, USA, 1984. 


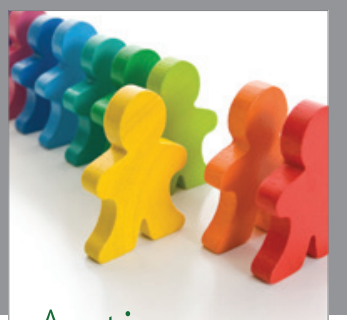

Autism

Research and Treatment
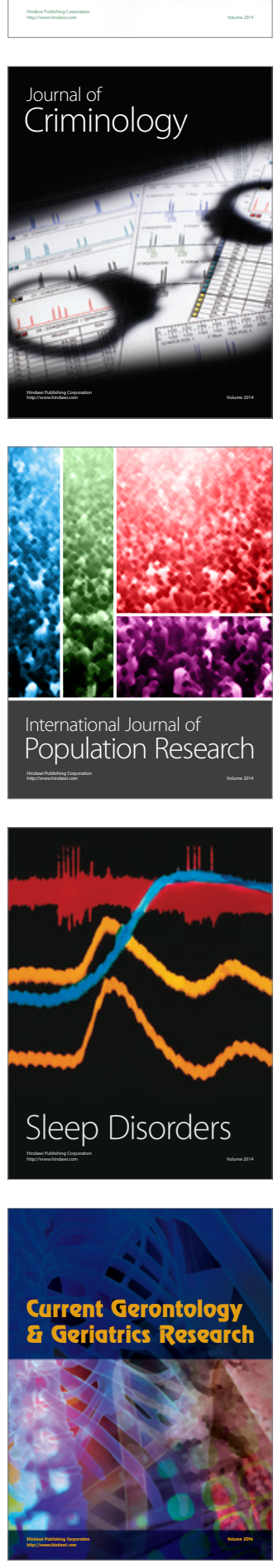
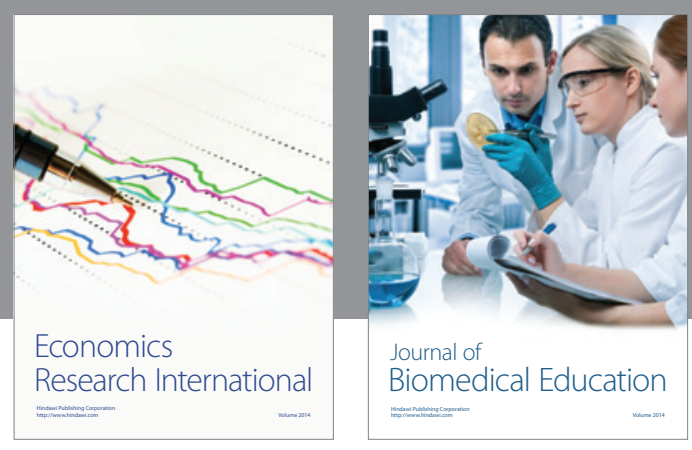

Journal of

Biomedical Education

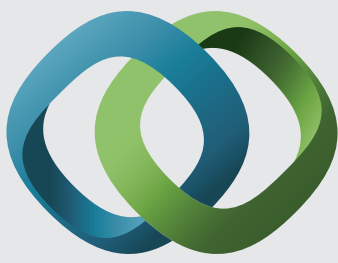

\section{Hindawi}

Submit your manuscripts at

http://www.hindawi.com
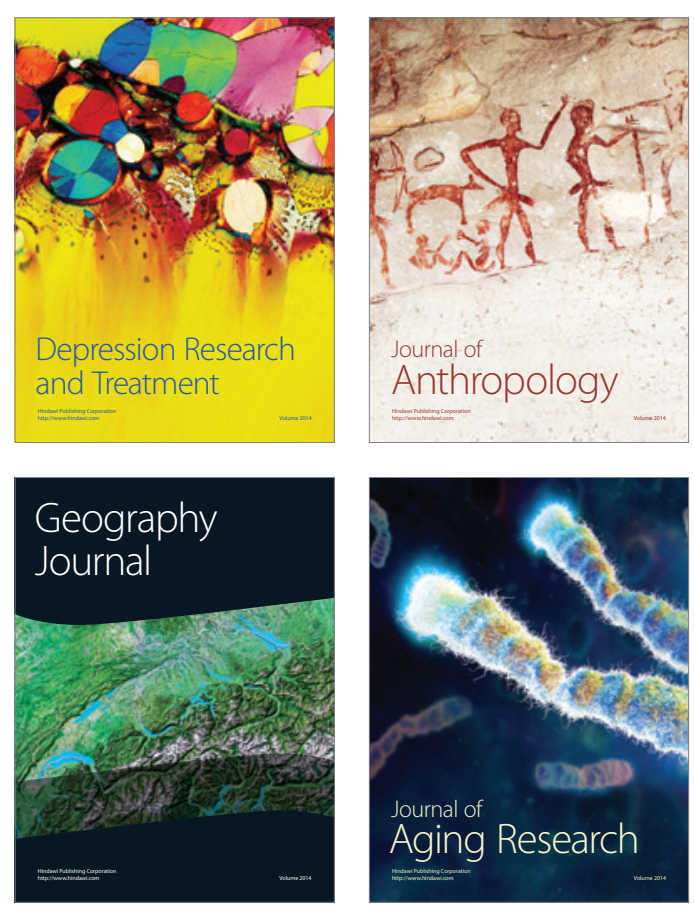

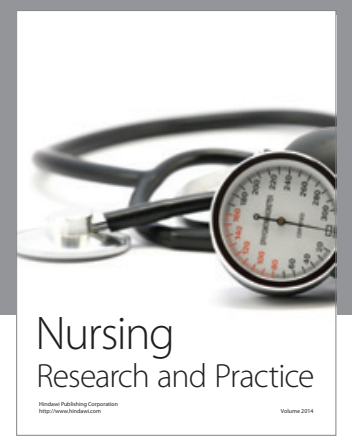

Nursing

Research and Practice

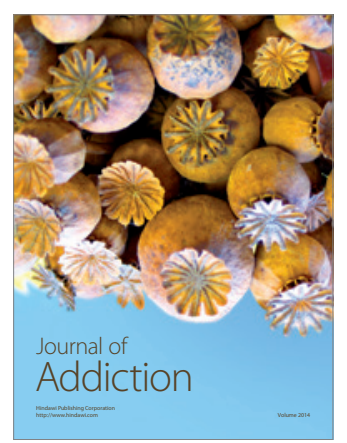

Child Development

Research

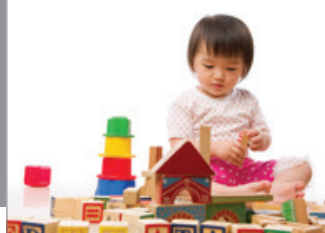

迥
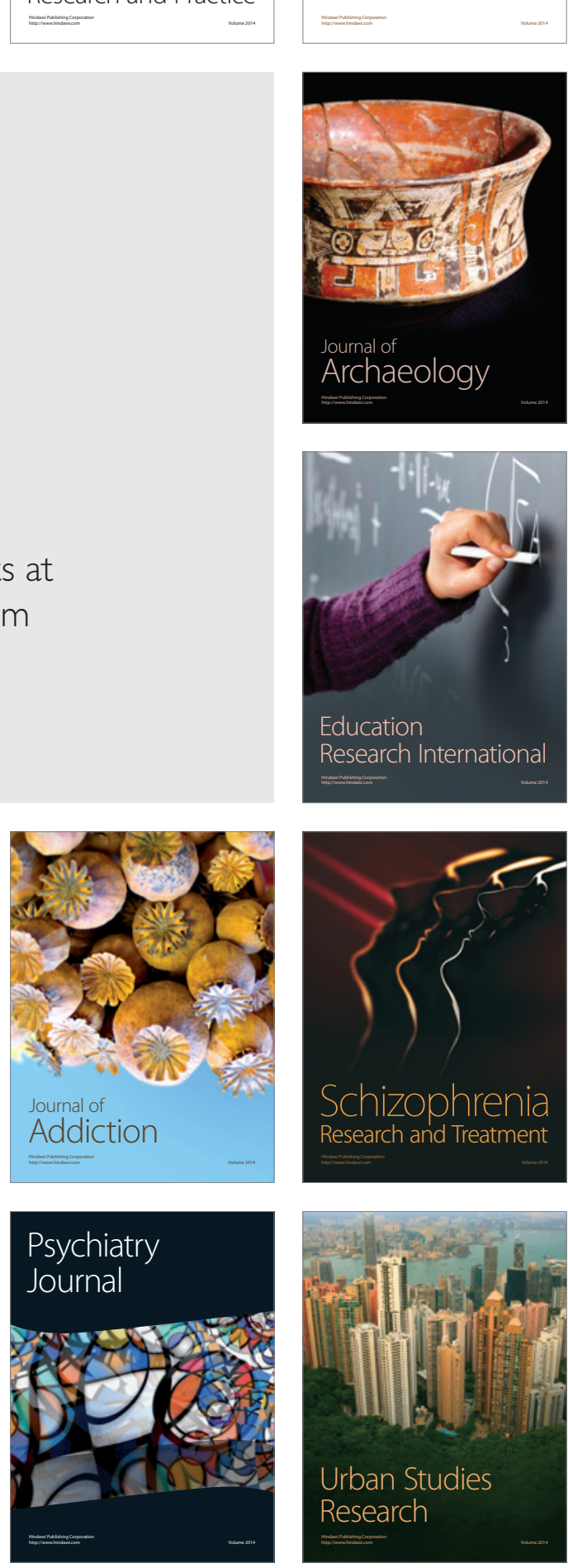